\title{
INTERVENÇÕES DE ENFERMAGEM PROMOTORAS DA ADAPTAÇÃO DA MULHER AO CANCRO DA MAMA
}

\author{
Ricardo Gil Silva \\ Mestre em Enfermagem Médico-Cirúrgica; Especialista em \\ Enfermagem Médico-Cirúrgica; AssistenteConvidado / \\ Instituto Português de Oncologia do Porto; \\ Escola Superior de Enfermagem do Porto \\ rgilsilva@gmail.com
}

\author{
Luís Miguel Ferreira \\ Professor Adjunto; Mestre em Ciências \\ de Enfermagem / Escola Superior de \\ Enfermagem do Porto \\ Imferreira@esenf.pt
}

\author{
Filipe Pereira \\ Professor Coordenador; Doutor em Ciências de \\ Enfermagem; Mestre em Ciências de Enfermagem / \\ Escola Superior de Enfermagem do Porto \\ filipereira@esenf.pt
}

RESUMO: Introdução: 0 cancro da mama é a doença oncológica com maior incidência na mulher. Identificar os elementos estruturantes das intervenções de enfermagem com vista a promover a adaptação da mulher ao cancro da mama é uma prioridade que carece de sistematização e proficiência para serem implementadas na prática clínica.

Metodologia: Revisão integrativa da literatura, de acordo com o modelo PVO (Paciente/População/ Problema, Variáveis em estudo e Outcomes/Resultados), entre os anos 2011 a 2016, com análise de 30 artigos através de um modelo de análise desenvolvido pelos investigadores que assenta em categorias indispensáveis ao desenvolvimento de intervenções de enfermagem baseadas em evidências.

Objetivos: Identificar os elementos estruturantes das intervenções de enfermagem com vista a promover a adaptação da mulher ao cancro da mama.

Resultados: A análise dos dados permitiu validar como referencial teórico dominante a Teoria do Autocuidado e, face às necessidades identificadas por esta população surge, em destaque, o sistema de apoio educativo. Nesta perspetiva de requisitos de desvio à saúde, as terapêuticas que se destacam são do tipo informar e educar - pretendem capacitar a mulher para a promoção das atividades de autocuidado.

Neste contexto e, atendendo ao anterior, as formas de implementação predominantes são sessões informativas e educativas, individuais e em grupo e com recurso a conteúdos disponíveis em suporte verbal e escrito. A utilização de novos recursos tecnológicos de informação e comunicação foi igualmente uma estratégia descrita mas os autores alertam que a utilização destes requer, nos seus utilizadores, altos níveis de motivação. 0 envolvimento de elementos significativos ou familiares foi igualmente destacado como recurso externo que pode determinar a adesão e envolvimento das mulheres ao plano terapêutico.

Conclusões: A identificação dos elementos estruturantes das intervenções de enfermagem, com vista a promover a adaptação da mulher ao cancro da mama, contribuirá para ajudar os enfermeiros dos contextos práticos, a conceberem e implementarem cuidados de enfermagem diferenciados no sentido de capacitar a mulher para que seja capaz de realizar todas as atividades inerentes ao autocuidado, de uma forma responsável e consciente.

PALAVRAS-CHAVE: Neoplasia mamária; Mulher; Enfermagem; Autocuidado. 
ABSTRACT:Introduction: Breast cancer is the oncological disease with higherincidence in women. Identify the structuring elements of nursing interventions to promoting the adaptation of women to breast cancer is a priority that lacks systematization and proficiency to be implemented in clinical practice.

Objectives: Identify the structuring elements of nursing interventions to promoting the adaptation of women to breast cancer.

Methodology: Integrative review of literature, according to the PVO model (Patient/Population/ Problem, Variables and Outcomes/Results), between the years 2011 to 2016, with analysis of 30 articles through an analysis model developed by investigators based in categories essential to the development of evidence-based nursing interventions.

Results: Data analysis made it possible to validate how dominant theoretical referential theory of Selfcare and, with regard to the needs identified by this population surge, highlighted, the educational support system. In this perspective of health deviation requirements, the therapies that stand out are to inform and educate and empower the woman aim to promote self-care activities. In this context and in view of the above, the predominant implementation forms are informational and educational sessions, individual and group and using content available in verbal and written support. The use of new information and communication technology resources was also a strategy described but the authors warn that the use of these requires, in its users, high levels of motivation. The involvement of significant elements or family was also highlighted as external resource that can determine the membership and involvement of women at therapeutic plan.

Conclusions: The identification of the structural elements of the nursing interventions to promote the adjustment of woman to breast cancer, will contribute to help nurses of practical contexts, to design and implement differentiated nursing care towards to enable the woman to be able to perform all activities inherent in self-care, responsibly and consciously.

KEYWORDS: Breast neoplasms, Woman, Nursing, Self-care.

\section{Introdução}

A doença oncológica mamária é, em Portugal e na Europa, o tumor com maior incidência na mulher ${ }^{1}$. As alterações que o tratamento para o cancro da mama acarretam, do ponto de vista físico, psicológico e social, desafiam as mulheres a desenvolverem processos de adaptação complexos. Nesta linha, torna-se clara a necessidade de identificar e disponibilizar cuidados orientados para a promoção desta adaptação das mulheres, com um acompanhamento de forma sistematizada e proficiente a desempenhar pelos enfermeiros.

A adaptação da mulher à doença oncológica mamária não se confina, como é frequente encontrar, a um conjunto de aspetos psicológicos. O termo adaptação engloba todos os processos que a mulher desenvolve e mobiliza, no sentido de lidar e integrar as mudanças decorrentes da sua nova condição. Nesta perspetiva, todas as atividades desenvolvidas pelos enfermeiros no sentido de ajudar as mulheres a viverem com a doença e a prevenirem complicações são atividades consideradas promotoras da adaptação.
A qualidade da assistência de enfermagem em Portugal, regulamentada pela Ordem dos Enfermeiros², assenta no princípio de satisfazer as necessidades humanas básicas e de aumentar a autonomia dos clientes nos diversos domínios do autocuidado, através de processos de aprendizagem que visam uma readaptação funcional e emocional. Neste contexto, a adaptação pode ser entendida como um resultado major da integração, por parte da mulher, da sua nova condição decorrente do cancro da mama o que pode ser visto como desígnio dos cuidados prestados pelos enfermeiros ${ }^{3}$.

A capacidade da pessoa se adaptar, nomeadamente do ponto de vista funcional, está intimamente relacionada com as limitações que esta apresenta decorrentes do evento que determinou mudanças nas suas capacidades de desempenho, em termos de autocuidado ${ }^{4}$. As ações de autocuidado, quer as universais quer aquelas decorrentes do desvio à saúde, são de grande importância no contexto dos cuidados de enfermagem, na medida em que promover o autocuidado pode ser, em última instância, promover a adapta- 
ção. Os requisitos universais de autocuidado são comuns a todos os seres humanos e estão inerentes aos processos quotidianos da vida. Os requisitos associados ao "desvio à saúde" reportam as atividades que o indivíduo desenvolve para gerir a doença e o seu tratamento.

O presente trabalho surge na sequência de um outro mais amplo desenvolvido por Ferreira ${ }^{5}$ que, ao longo de várias etapas, desenvolveu um estudo qualitativo e um outro de perfil quantitativo, onde se identificou um conjunto de vinte e três (23) intervenções de enfermagem (IE) muito relevantes para efeitos de promoção da adaptação da mulher ao cancro da mama, consensualizadas por um painel com cerca de cento e quarenta (140) enfermeiros que exercem funções em centros de referências em oncologia. São exemplos dessas intervenções: Ensinar estratégias para diminuir o linfedema; Incentivar a autonomia no autocuidado; Envolver a pessoa no plano de cuidados; Ensinar estratégias para prevenir complicações/efeitos secundários do tratamento (cirurgia, quimioterapia, radioterapia, hormonoterapia); Ensinar sobre adequação no autocuidado; Instruir técnica de exercícios do membro afectado; entre outras.

Da leitura dos anunciados apresentados, percebe-se que a larga maioria das intervenções se centram na promoção das capacidades de autocuidado e na prevenção de complicações decorrentes do tratamento. A intencionalidade destas intervenções remetem para o conceito de "autocuidado" nos termos que nos é proposto por Orem Efectivamente, o autocuidado é o desenvolvimento de atividades executadas pela pessoa em seu próprio benefício, para a manutenção da vida e do seu bem-estar. Estas atividades dizem respeito às capacidades e habilidades para "tratar do que é necessário para se manter; manter-se operacional e lidar com as necessidades individuais básicas e íntimas e as atividades da vida diária”" (p.12).

O teor das intervenções que emergiram como "muito relevantes" na investigação de Ferreira ${ }^{5}$, à luz do modelo de $\mathrm{Orem}^{4}$, orientam-se para a promoção dos requisitos universais de autocuidado e de desvio à saúde. Assim, entendemos como necessário evoluir em termos de proficiência e sistematização das intervenções, para que as mesmas possam, efetivamente, ser implementadas pelos enfermeiros na prática clínica sempre com o enfoque na intenção terapêutica, como abordado por Meleis ${ }^{7}$ quando no aborda sobre o caráter prático da disciplina de enfermagem.
A delimitação dos elementos estruturantes das IE, de acordo com aquilo que é claro no seio da disciplina, remete para a necessidade de clarificar qual o referencial teórico das intervenções; qual o seu conteúdo/atividades; quais as formas como devem ser implementadas e; por exemplo, como deve ser ponderada a sua efetividade.

Com este trabalho pretendemos contribuir para a melhoria das práticas de enfermagem neste contexto específico através da identificação dos elementos estruturantes das intervenções de enfermagem mais relevantes para efeitos da promoção da adaptação das mulheres ao cancro da mama.

Do percurso realizado por Ferreira ${ }^{5}$ percebe-se que uma parte substantiva da assistência de enfermagem, face às mulheres com cancro da mama, se centra em focos de atenção direcionados para o autocuidado (requisitos universais e de desvio à saúde/gestão do regime terapêutico) perseguindo objetivos orientados para o desenvolvimento de "capacidades de autocuidado". Daqui resulta claro que a adaptação está, também, definida numa lógica de "resultado" centrado em capacidades funcionais e de desempenho das clientes. Com efeito, no contexto deste nosso percurso, concebemos a adaptação como capacidades e ações intencionais da cliente para "gerir novas situações" (p.3). Importa salientar que, na mesma classificação de enfermagem a que aludimos, tais ações se orientam, também, para "gerir o stresse e ter uma sensação de controlo e de maior conforto psicológico”' (p.34). É comum na literatura de enfermagem a apresentação da doença oncológica como altamente "stressante". Concordamos, na medida em que, reconhecidamente, a mulher com doença oncológica da mama experimenta "sentimentos de estar sob pressão e ansiedade ao ponto de ser incapaz de funcionar de forma adequada física e mentalmente; sentimento de desconforto; associado a experiências desagradáveis; associado à dor; sentimento de estar física e mentalmente cansado; distúrbio do estado mental e físico..." ${ }^{\prime 6}$ (p.121).

\section{Materiais e métodos}

Dos métodos de revisão da literatura existentes, a Revisão Integrativa da Literatura (RIL) surge como um dos que "melhor se adequa" à incorporação das evidências disponíveis na prática de enfermagem $8,9,10,11,12,13$.

Tendo como objetivo identificar as IE promotoras da adaptação das mulheres ao cancro da mama, delimitamos 
a seguinte questão de partida "Quais as intervençôes de enfermagem capazes de promover a adaptação das mulheres ao cancro da mama?". Como pretendíamos produzir uma síntese mais integrativa e não proceder a comparações (como tradicionalmente utilizado com o método $\mathrm{PICO}^{14}$ ), utilizamos o modelo ou estratégia PVO (Paciente/População/ Problema, Variáveis em estudo e Outcomes/Resultados) para formulação da questão de partida. A nossa População foram mulheres com neoplasia da mama, as Variáveis em estudo as intervenções de enfermagem e os Resultados relativos à adaptação (enquanto resultado).

Delimitada a temática de pesquisa, e mantendo sempre o foco no objetivo da nossa pesquisa, procedeu-se ao estabelecimento dos critérios de inclusão que os estudos teriam de cumprir para integrarem o nosso "corpus de análise": evidências recentes, publicadas nos últimos cinco anos (entre 2011 e 2016); focadas em clientes com um perfil semelhante ao das clientes assistidas na realidade nas "Clínicas de Mama" nos centros de referência em oncologia (mulheres, com idade superior a 18 anos e com o diagnóstico médico principal de neoplasia da mama); e presença dos descritores no título ou no resumo ou nas palavras-chave/ termos do assunto.

Procedemos à extração do material entre os dias 16 de março e 14 de abril de 2016, em todas as bases de dados electrónicas presentes nos agregadores de bases de dados EBSCOhost Web, Scopus e Web of Science ${ }^{\mathrm{TM}}$, a que tivemos acesso via Escola Superior de Enfermagem do Porto.

A identificação dos descritores a serem usados é crucial para este tipo de revisão da literatura. Recorremos aos MeSH terms da National Library of Medicine dos Estados Unidos da América. Esta nossa opção radicou na amplitude, reconhecimento e utilização que este sistema de “indexação de conteúdos” tem à escala global. Assumimos que, na procura e identificação dos descritores $\mathrm{MeSH}$ com relevância para este estudo, foi nossa preocupação integrar, sempre que possível, descritores ascendentes e descendentes dos termos a incluir, de forma a alargar o alcance da pesquisa. Contudo, existiram termos que aparentemente poderiam ser incluídos na nossa pesquisa mas, após leitura do "scope note", verificámos que não se integravam dentro das nossas categorias de pesquisa.

Do conjunto das 23 IE identificadas como muito relevantes pelos enfermeiros no estudo de Ferreira ${ }^{5}$ emergem cinco grandes intencionalidades: 1) Promover capacidades de desempenho nos requisitos universais do autocuidado; 2) Promover capacidades para gerir o regime terapêutico; 3) Promover conhecimento e capacidade para prevenir efeitos secundários e complicações dos tratamentos; 4) Promover conhecimento e capacidade para prevenir/controlar linfedema e; 5) Promover o envolvimento da família e consciência sobre mudanças. Destas, identificámos descritores (MeSH terms) centrados em algumas áreas específicas, como: equipamentos de apoio no autocuidado; consciencialização das mulheres sobre as suas limitações no autocuidado; gestão do regime terapêutico; envolvimento da família; e prevenção de complicações. Os descritores identificados foram utilizados na composição das frases booleanas, colocando com restrição o facto dos mesmos estarem presentes no título ou no resumo ou nas palavras-chave/termos do assunto. No sentido de tornar mais ágil a busca de material relevante, decidimos compor cinco frases booleanas e não apenas uma. Cada uma das cinco frases booleanas incluía as quatro componentes centrais dos nossos interesses - Cancro da Mama ("Breast Neoplasm"); Enfermagem (“Nurs"); Mulheres ("Women”); e Autocuidado ("Self Care") - associado a um outro componente relativo a: Equipamento ("Equipment and Supplies"); Consciencialização (“Awareness"); Envolvimento pessoal ("Patient participation”); Envolvimento da Família ("Famil"”); e Complicações (“complication").

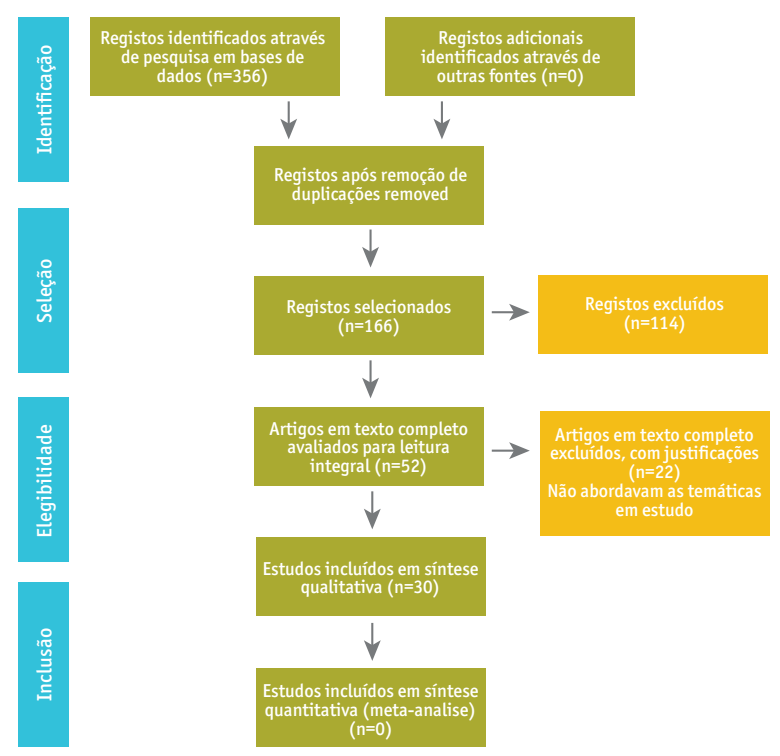

Figura 1. Fluxograma baseado no modelo PRISMA ${ }^{15} \mathrm{com}$ procedimento de seleção dos estudos 
Deste processo de seleção (figura 1) foram reunidas 30 referências ${ }^{16-45}$ que constituíram o nosso "corpus de análise”. Estes artigos foram analisados e sujeitos a extração de dados através de um modelo elaborado pelos investigadores visto o desenvolvimento e análise das IE estar ainda pouco explorado na literatura da disciplina.

Neste sentido, recorremos a dois modelos de desenvolvimento de IE baseadas em evidências. $\mathrm{O}$ primeiro, sugerido por Meijel e colaboradores ${ }^{46}$, e o segundo, proposto por Aranda ${ }^{47}$. Estes dois modelos, dada a sua convergência em termos de conceitos estruturantes e foco na enfermagem, serviram-nos para delimitar as categorias a adotar na análise dos 30 artigos reunidos. Ambos os modelos destacam como fundamental a inclusão da opinião dos enfermeiros "da prática" acerca da relevância, utilidade clínica e aplicabilidade das intervenções. Sugerem ainda, que a visão dos beneficiários das intervenções é essencial, no sentido de perceber, entre outros aspetos, os seus "resultados".

Ambos os modelos estruturam e descrevem um processo sistematizado e rigoroso para o desenvolvimento e revisão de IE baseadas em evidência e partilham entre si um conjunto de critérios que deve guiar todo o processo de análise das intervenções. Partindo dos elementos estruturantes destes modelos, e considerando a sua utilidade para efeitos do processo de análise ao material emergente da pesquisa realizada, definimos oito categorias do "nosso modelo de análise" que, à partida, foi definido como alicerce deste processo:

1) Referenciais Teóricos das Intervenções;

2) População Alvo, Beneficiários/Clientes das Intervenções;

3) Conteúdo das Intervenções;

4) Estratégias de Implementação das Intervenções;

5) Complexidade das Intervenções;

6) Integridade das Intervenções;

7) "Dose Terapêutica" das Intervenções;

8) Validade das Intervenções e Medidas de Resultado.

O processo de seleção e análise dos estudos foi efetuado por dois revisores, de uma forma independente, nomeadamente pelo investigador principal do estudo e por um dos investigadores secundários. Os artigos foram selecionados de acordo com as temáticas que ocupavam o nosso estudo e as opções de exclusão radicaram no mesmo princípio, artigos que não se centravam nas temáticas em questão com a presente investigação. Importa salientar que todos os arti- gos reunidos nesta análise foram publicados em revistas com arbitragem científica e análise por painel de revisores/ peritos.

\section{Resultados}

Dos 30 artigos reunidos ${ }^{16-45}$, constatámos que 24 arti$\operatorname{gos}^{16-18,20,22,24,25,27-31,33-35,37-45}$ reportam, de forma explícita ou implícita, IE. Os seis artigos restantes ${ }^{19,21,23,26,32,36}$, não se centrando em intervenções propriamente ditas, foram incluídos na análise porque fazem referência a aspetos que podem ser enquadrados nos "modos e estratégias" de implementação e "avaliação" das IE.

Da análise efetuada não encontramos descritos enunciados e/ou sintaxes de intervenções como habitualmente se encontram disponíveis nos sistemas de informação. Conseguimos, sim, identificar aspetos relativos à natureza das intervenções, o que nos pareceu adequado, neste ponto, recorrer à estrutura da Norma ISO 18104 proposta pela International Organization for Standardization ${ }^{48}$. A referida norma apresenta-nos como categorias: Ação; Alvo; Meio; Via; Local e Recetor (Beneficiário) dos cuidados.

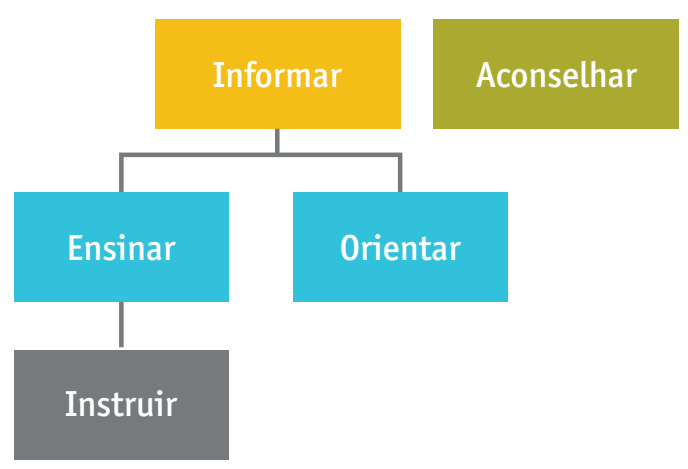

Figura 2. Tipos de ação das intervenções de enfermagem reportadas nos artigos analisados

No que diz respeito aos tipos de ação, as IE reportadas nos artigos reunidos inscrevem-se fundamentalmente no âmbito do Informar - "Comunicar alguma coisa a alguém" ${ }^{49}$ (p.97) e, dentro destas, do tipo "Ensinar" e "Instruir"; - "Dar informação sistematizada a alguém sobre temas relacionados com a saúde" ${ }^{49}$ (p.96) e - "Fornecer informação sistematizada a alguém sobre como fazer alguma coisa"49 (p.97), respetivamente. Estas intervenções têm como objetivo desenvolver competências de cariz essencialmente cognitivo e habilidades de cariz mais 
instrumental. Estes tipos de ação, que se vislumbram na grande maioria das intervenções apresentadas pelos diferentes autores, têm por base a intenção terapêutica de capacitar as clientes para lidarem com os desafios de saúde que o cancro da mama lhes impõe. Algumas das IE incluídas nos artigos tomam por tipo de ação o "Orientar" ou o "Aconselhar". Qualquer um destes tipos de ação, na Classificação Internacional para a Prática de Enfermagem (CIPE), são especificações do "Informar" e visam "dirigir alguém para uma decisão em assuntos relacionados com a saúde" 49 (p.98). Também aqui se percebe que, a intenção subjacente às intervenções que usam estes dois últimos tipos de ação, radica na preparação e aumento do repertório de recursos pessoais das clientes para se adaptarem, o mesmo é dizer: viverem uma transição no rumo da "saúde" ${ }^{50}$.

\section{Evidências emergentes}

\section{1) Referenciais Teóricos das Intervençôes}

Dezassete dos 30 artigos analisados fazem referência a uma teoria ou modelo concetual que está na base da sua intenção terapêutica. Destes, oito artigos ${ }^{16-18,24,27,35,38,41}$ baseiam-se no modelo de Autocuidado proposto por Orem. Estes artigos colocam a tónica nos "requisitos de desvio à saúde".

\section{2) População Alvo, Beneficiários/Clientes das Intervençôes}

O conceito de "recetor dos cuidados", incluído na norma ISO 1810448, é enquadrável nesta nossa categoria de análise. Todos os artigos incluídos nesta revisão fazem referência à população alvo das intervenções. O elemento que estrutura e nos dá referências para os diferentes beneficiários radica na fase do percurso terapêutico em que as mulheres se encontram. Cerca de dois terços dos artigos centram-se em mulheres que se encontram no período após a cirurgia: dez artigos ${ }^{18,19,22,24,25,27,35,36,41,43}$ estão focados em mulheres com linfedema (real ou potencial); nove $\operatorname{artigos}^{17,23,26,30-33,42,45}$ estão focados em mulheres sujeitas a tratamento de quimioterapia (QT) e/ou radioterapia (RT) e; quatro artigos ${ }^{28,29,38,44}$ tomam por beneficiários das intervenções mulheres que já se encontram no período de follow-up. Seis artigos ${ }^{16,20,21,37,39,40}$ centram-se, essencialmente, em mulheres em fases iniciais do tratamento da doença, que aguardam cirurgia ou vão iniciar tratamento de QT ou RT.

3) Conteúdo das Intervenções

Atendendo ao facto da larga maioria das intervenções referidas nos artigos reunidos nesta revisão serem do tipo "Informar" ${ }^{49}$, o conteúdo das intervenções reporta-se ao teor da informação daquelas ações. O conceito de conteúdo das intervenções é sobreponível à categoria "alvo das intervenções” da norma ISO 1810448. Aqui, os alvos são importantes na medida em que, dizendo respeito “... às entidades que são afetadas pela ação ou que lhe dão conteúdo...", permitem-nos ter uma noção clara do conteúdo das intervenções que são fundamentalmente do tipo apoio-educativo ${ }^{4}$.

Quando se analisam “... as entidades que são afetadas pela ação ou que lhe dão conteúdo...” - Alvo ${ }^{48}$, no conjunto dos artigos reunidos identificam-se oito alvos distintos, aqui apresentados, de forma decrescente, em função da sua frequência: Tratamentos; Doença ou Processo patológico; Linfedema (Edema); Efeitos secundários (dos tratamentos); Complicações (dos tratamentos); Gestão do regime medicamentoso; Atividades diárias; e Apoio (familiar ou social). Os conteúdos mais frequentes referem-se, fundamentalmente, a informação sobre os tratamentos, ao processo patológico/doença e aos efeitos secundários da medicação. A prevenção, controlo e auto-vigilância do linfedema, a par de outras consequências dos tratamentos médicos, assumem lugar de destaque.

A gestão do regime terapêutico, concretamente a gestão do regime medicamentoso, nestas clientes, é um aspeto crucial, o que justifica a pertinência de vários autores a orientarem o conteúdo dos seus estudos para esta área.

\section{4) Estratégias de Implementação das Intervenções}

Esta categoria pretende responder à questão: "Como devem ser educadas e apoiadas estas clientes, de forma a promover o seu autocuidado?". Existem 24 referências $^{16-18,20,22,24,25,27-31,33-35,37-45}$ à utilização de estratégias presenciais e oito referências a estratégias à distância $^{16,17,20,28,29,34,37,39}$. As estratégias à distância são muito adequadas para promover o envolvimento das mulheres no plano terapêutico e também para desenvolver competências de gestão do regime terapêutico, em particular, medicamentoso. Contudo, de acordo com Kuijpers e colaboradores $^{29}$, a utilização desta estratégia, carece de altos níveis de motivação por parte das mulheres, o que requer ponderação do enfermeiro na utilização deste tipo de estratégias.

Ainda nesta categoria, percebe-se a existência de estratégias educacionais do tipo "individual" e outras baseadas "em grupos". Existem 17 referências quer a implementa- 
ção de estratégias do tipo individual $1^{16,22,24,25,27-29,31,33,35,38,40-45}$, quer em grupos ${ }^{16-18,20,24,25,27-30,34,35,37,39,41-43}$. Nas abordagens em grupo, estão contemplados os grupos de "ajuda" entre pares (mulheres com cancro da mama), muito valorizadas pelas mulheres ${ }^{20,27,34,37}$. O envolvimento de elementos familiares/significativos é valorizado por alguns autores, referindo que aumentam a adesão das mulheres ao plano de tratamento e ressalvam a importância de incluir estes elementos no plano terapêutico ${ }^{25,28,42,44}$.

O recurso a tecnologias de informação e comunicação é reportado por vários autores ${ }^{16,17,20,29,34,37,39}$ sendo uma estratégia associada a intervenções do tipo informar/ensinar sobre doença (sinais, sintomas, fatores de risco, ...); os tratamentos disponíveis; os efeitos secundários dos tratamentos; as possíveis complicações; estratégias de reabilitação...

Oito artigos sublinham a importância de combinar a informação verbal com um suporte escrito de cariz complementar, de forma a reforçar a informação disponibili$\operatorname{zada}^{16-18,20,34,40,41,44}$.

\section{5) Complexidade das Intervenções}

Dos 24 artigos que fazem referência a $\mathrm{IE}^{16-18,20,22,24,25,27-}$ 31,33-35,37-45, todos se referem a intervenções do tipo complexo. Não existem, nos artigos analisados, referências a intervenções "simples".

\section{6) Integridade das Intervenções}

Das intervenções identificadas nos artigos analisados, verificámos que em 14 casos há alguma referência à sequência de implementação das diferentes atividades das intervenções ${ }^{16-18,20,24,27,28,30,34,37,38,41,43,45}$. Todavia, o grau de estruturação apresentado não é suficientemente preciso para que nos seja possível falar em "roteiro". As referências disponíveis nos artigos são muito genéricas. A título de exemplo, vemos orientações que remetem para a necessidade (óbvia) da educação referente à cirurgia dever ser realizada, preferencialmente, antes da mesma ocorrer, para que a mulher tenha tempo de assimilar a informação referente à mesma e para que possa ter tempo de colocar as respetivas dúvidas e receios (6 referências). Os efeitos secundários e/ou possíveis complicações dos tratamentos devem, igualmente, ser abordados antes destes ocorrerem (8 referências).

\section{7) "Dose Terapêutica" das Intervenções}

Apenas em dez casos há referências a aspetos relacionados com a "dose terapêutica" das intervenções ${ }^{16,17,18,20,22,25,28,30,40,43}$. São abordados aspetos relaciona- dos com o número de sessões educativas, duração média de cada sessão e momentos em que estas devem ocorrer, nomeadamente antes, durante e após os tratamentos. Recomenda-se um maior número de sessões educativas quando se pretende o reforço da informação com vista a consciencializar as mulheres sobre a sua nova condição e para que possam, de uma forma consciente e responsável, fazer uma autogestão do regime terapêutico ${ }^{16,19,20,25,30,40}$. A duração das sessões varia entre 40 e 150 minutos. As sessões mais demoradas são aquelas que pretendem capacitar as mulheres para efetuarem uma autogestão eficaz do seu regime terapêutico, após tratamento ${ }^{28,30}$. Tratam-se de intervenções que incorporam conteúdos instrumentais e visam o desenvolvimento de habilidades das mulheres.

8) Validade das Intervençôes e Medidas de Resultado

Vinte e quatro artigos ${ }^{16-23,25-30,32,34,35,37,39-43,45}$ fazem referência ao resultado que pretendem atingir com as intervenções apresentadas e referem formas de verificar as mudanças verificadas. No âmbito das intervenções reunidas, de acordo com os autores consultados, a forma mais adequada de verificar se o objetivo proposto foi, efetivamente, alcançado, é através da observação/demonstração/ validação da aquisição das competências pretendidas por parte das mulheres. Os artigos que explicitam os resultados pretendidos fazem-no sobre a forma de "critérios de resultado". São exemplos de critérios de resultado: "Identifica os efeitos secundários do tratamento"; "Realiza os exercícios de prevenção de linfedema"; "Conhece as formas de prevenir as complicações dos tratamentos". Com frequência, os diferentes artigos remetem para resultados ou medidas de avaliação da eficácia das intervenções em aspetos mais genéricos, ainda que medidos com as "escalas" habituais, como: diminuição dos níveis de ansiedade e stress.

Para além deste tipo de medidas de validade das intervenções, vários autores remetem para as "opiniões das clientes". Aqui destacam-se aspetos como, por exemplo, o aumento da perceção de auto-eficácia para gerir os efeitos secundários da medicação. Verificamos, ainda referências a opiniões como: "sentir-se mais preparada para encarar o tratamento"; "sentir-se melhor consigo própria”; ou "sentir-se mais confiante, quando dispõe de apoio de familiares ou outros significativos".

Relativamente à opinião dos enfermeiros que implementam as intervenções na prática e também dos beneficiários das mesmas, as referências analisadas não apresentam, 
explicitamente, uma opinião ou informação especificamente relacionada com uma intervenção em concreto.

\section{Discussão}

Os resultados emergentes da análise efetuada aos artigos selecionados permitem identificar e sintetizar um corpo de conhecimentos que pode orientar a prática dos enfermeiros neste contexto assistencial.

O modelo concetual proposto Orem é suficientemente abrangente para nos ajudar a explicar as necessidades em cuidados de enfermagem destas mulheres, assim como o tipo de ajuda profissional a ser disponibilizada pelos enfermeiros. As intervenções baseadas neste referencial teórico, quando inscritas num sistema do tipo "apoio-educativo", visam ajudar as clientes a aumentarem os seus recursos para tomarem iniciativas e assumirem responsabilidades pelo seu próprio cuidado e saúde. A natureza das intervenções inferidas dos artigos inscreve-se numa lógica de apoio e educação, tendo em vista satisfazer requisitos de autocuidado relacionados com o desvio à saúde.

As intervenções descritas na literatura tomam por clientes, fundamentalmente, mulheres em tratamento ativo, em particular cirúrgico, destacando este subgrupo como alvo prioritário da atenção dos enfermeiros.

Podemos inferir que as intervenções disponíveis nos artigos reunidos tomam por "grandes" alvos: o conhecimento das clientes sobre tratamentos, efeitos secundários da medicação, entre outros; e as capacidades para, por exemplo, prevenir e controlar complicações dos tratamentos e ainda, prevenir e controlar o linfedema. Aqui percebe-se, de alguma forma, uma orientação para a promoção de competências cognitivas das clientes, mas também de capacidades instrumentais, de que são exemplo as "técnicas de exercício muscular e articular".

A possibilidade de estabelecer contacto direto com enfermeiros que integram a equipa assistencial, mostra-se uma estratégia muito valiosa. Esta informação sublinha a importância dos enfermeiros privilegiarem a implementação de estratégias presenciais.

O recurso a estratégias tecnológicas mais recentes desperta na nossa comunidade uma área que merece investimento, para que possamos promover o acompanhamento a estas clientes. $\mathrm{O}$ avanço tecnológico disponibiliza a esta população material informativo nos mais diversos formatos. Promover o envolvimento e adesão destas mulheres às "novas tecnologias" pode mostrar-se uma ferramenta muito poderosa em termos de adaptação. Carece, contudo, de um aconselhamento adequado para que a informação a ser disponibilizada e acedida pelas mulheres seja adequada e verosímil.

Com estes achados podemos inferir que as IE desenvolvidas com a intenção de assistir as mulheres com cancro da mama, no decurso da doença e dos tratamentos, se centram em dar resposta a vários resultados e contemplam vários elementos de ação, exigindo, por parte dos enfermeiros, uma visão focalizada e crítica nas necessidades em concreto destas mulheres. De salientar que a evidência disponível nos artigos reunidos nesta revisão sobre a complexidade das intervenções é limitada.

Como se percebe, o aspeto das intervenções relacionado com a integridade das mesmas, surge de uma forma muito incipiente na literatura, pelo que, desde logo, se percebe que a evidência disponível, a este respeito, é muito fraca. A sequência de implementação dos vários elementos de ação deve ser cuidadosamente determinada para que a finalidade da intervenção seja alcançada. Urge assim, investigar sobre quais os melhores roteiros de implementação destas intervenções complexas.

A adequação da "intensidade" da intervenção deve, na lógica de conceção do enfermeiro, responder às necessidades, expectativas e caraterísticas individuais de cada mulher, devendo, assim, ser cuidada. Não há evidências sólidas, nos artigos reunidos, sobre qual a "intensidade" das intervenções. Uma vez mais, esta deve ser ajustada à finalidade da intervenção.

Quanto aos momentos adequados para a realização das intervenções, percebe-se a prevalência de uma abordagem do tipo "antecipatório" à experiência, por parte da cliente. De ressalvar que existem aspetos determinantes que devem ser atendidos pelos enfermeiros que acompanham estas mulheres, nomeadamente "o intervir o mais precoce possível" 43 e informar e/ou educar "previamente ao tratamento" 22 , para que as mulheres se sintam preparadas para lidarem com os diferentes desafios que experienciam ao longo das diferentes fases dos "tratamentos".

A determinação de formas de verificar e medir os resultados pretendidos é tão determinante quanto o objetivo do mesmo. Nos artigos analisados a forma mais usual de verificar o resultado da intervenção é através da validação/ observação do mesmo. Neste aspeto, a opinião do bene- 
ficiário da intervenção sobre o que mudou mas também a experiência do enfermeiro enquanto prescritor e implementador da intervenção são dados válidos que permitem estabelecer a validade dessa mesma intervenção.

\section{Conclusão}

Os planos assistenciais de enfermagem têm vindo a sofrer alterações ao longo do tempo, em função dos (novos) desafios assistenciais e, fundamentalmente, das evidências que se vão consolidando, fruto da prática e investigação dos enfermeiros.

A teoria do autocuidado de Orem é um referencial muito válido para o desenho e implementação daquelas intervenções, o que mostra que, numa lógica de enfermagem, promover o autocuidado é promover a adaptação. As competências de autocuidado remetem, essencialmente, para a mestria ${ }^{50} \mathrm{e}$ a adaptação para o resultado final do processo (saudável) de transição. A natureza das intervenções reunidas inscreve-se num sistema de apoio e educação. $\mathrm{O}$ conteúdo das intervenções reunidas está bem definido. Podemos dizer que há suficiente evidência sobre "o que ensinar e instruir”, para além destes conteúdos serem largamente consensuais, atravessando vários artigos reunidos. A incorporação, numa lógica complementar, de estratégias de implementação das intervenções com base nas tecnologias de informação e comunicação é recomendada e sustentada nas evidências emergentes. Urge lançar mão de abordagens "à distância", assim, como a abordagens com grupos de pares (outras doentes) e com os familiares.

Os roteiros ou guias de implementação das diferentes atividades das intervenções, dada a sua (das intervenções) complexidade, não são claros, pelo que daqui emerge como necessário criar, pelo menos à escala local, consensos para a prática.

Os artigos incluídos nesta Revisão da Literatura tornam clara a necessidade de implementar as intervenções identificadas de uma forma antecipatória "à necessidade" da mulher. A lógica antecipatória preconizada é congruente com o conceito de "preparação e conhecimento" antecipatório, proposto por Meleis e colaboradoras ${ }^{50}$. Apostar na preparação e conhecimento (antecipatório) das mulheres é uma "condição facilitadora" do curso da transição/adaptação. As medidas de resultado das intervenções podem ser vistas, neste conjunto de intervenções, a dois níveis. O primeiro, numa linha alicerçada em "critérios de resultado" (mais objetivos), partilhados por todos os enfermeiros envolvidos no plano assistencial, critérios que radicam nos próprios dados necessários à identificação dos diagnósticos de enfermagem que justificam cada uma das intervenções. O segundo nível, em que os resultados das intervenções devem ser vistos remete para "medidas" mais genéricas, derivadas da opinião/satisfação das clientes com as intervenções implementadas.

Destes factos somos levados a considerar que, implementando intervenções de enfermagem dirigidas a estes objetivos, estamos a levar por diante o trabalho "mais relevante" para promover a adaptação das mulheres ao cancro da mama. A revisão integrativa da literatura aqui relatada faz a síntese de alguns aspetos que nos podem ajudar a fazer aquele "trabalho relevante".

\section{Referências bibliográficas}

1. Registo Oncológico Nacional. (2016). Registo Oncológico Nacional 2010. Porto: Instituto Português de Oncologia do Porto Francisco Gentil - EPE, ed. Porto, 144 p.

2. Ordem dos Enfermeiros. (2001). Divulgar: Padrões de qualidade dos cuidados de enfermagem. Consultado em Maio, 2015, em http://www.ordemenfermeiros.pt/publicacoes/Documents/ divulgar\%20-\%20padroes $\% 20$ de $\% 20$ qualidade $\% 20$ dos $\% 20$ cuidados.pdf

3. Roy, C. \& Andrews, H. A. (2001). Teoria da enfermagem. 0 modelo de adaptação de Roy. Lisboa: Instituto Piaget.

4. Orem, D. (2001). Nursing: Concepts of practice. St. Louis: Mosby, 2001.

5. Ferreira, L. M. R. (2014) Terapêuticas de enfermagem promotoras da adaptação em mulheres com cancro da mama. Porto:

Universidade Católica Portuguesa - Instituto de Ciências da Saúde - Porto, 375p. Tese de doutoramento.

6. International Council of Nurses. (2015). CIPE® Versão 2015 Classificação Internacional para a Prática de Enfermagem. Lisboa: Ordem dos Enfermeiros.

7. Meleis, A. I. (2005). Theoretical Nursing: Development and Progress. Philadelphia: 3a Ed., Lippincott Williams \& Wilkins.

8. Mendes, K., Silveira, R. C. \& Galvão, C. M. (2008). Revisão integrativa: método de pesquisa para a incorporação de evidências na saúde e na enfermagem. Texto Contexto Enferm,17(4), 758-764.

9. Whittemore, R. (2005). Combining Evidence in Research. Nursing Research, 54(1), 56-62.

10. Whittemore, R. \& Knafl, K. (2005). The integrative review: Updated methodology. Journal of Advanced Nursing, 52(5), 546-553.

11. Broome, M. E. (2000). Integrative literature reviews for the development of concepts. In Concept Development in Nursing, 2nd ed (Rodgers B.L. \& Knafl K.A., eds), W.B. Saunders Co., Philadelphia, PA. July, 231-250.

12. Roman, A. \& Friedlander, M. R. (1998). Revisão integrativa de pesquisa aplicada à enfermagem. Cogitare enferm, 3(2), 109-112.

13. Ganong, L. H. (1987). Integrative reviews of nursing research. Research in Nursing \& Health, 10(1), 1-11.

14. Santos, C. M., Pimenta, C. A. \& Nobre, M. R. (2007). A estratégia PICO para a construção da pergunta de pesquisa e busca de evidências. Rev Latino-am Enfermagem, 15(3), 2-5.

15. Moher, D., Liberati, A., Tetzlaff, J. \& Altman, D. G. (2009). Preferred reporting items for systematic reviews and metaanalyses: the PRISMA statement. BMJ, 339, b2525.

16. Aranda, S., Jefford, M., Yates, P., Gough, K., Seymour, J, Francis, P., Baravelli, C., Breen, S. \& Schofield, P. (2012). Impact of a novel nurse-led prechemotherapy education intervention 
(ChemoEd) on patient distress, symptom burden, and treatment-related information and support needs: Results from a randomised, controlled trial. Annals of Oncology, 23(1), 222-231.

17. Børøsund, E., Cvancarova, M., Moore, S. M., Ekstedt, M. \& Ruland, C. M. (2014). Comparing effects in regular practice of e-communication and Web-based self-management support among breast cancer patients: preliminary results from a randomized controlled trial. Journal of medical Internet research, 16(12), e295. Consultado em Março, 2016 em http://www.jmir. org/2014/12/e295/

18. Cho, H. S. M., Davis, G. C., Paek, J. E., Rao, R., Zhao, H., Xie, X.-J., Yousef, M. J., Fedric, T., Euhus, D. H. \& Leitch, M. (2013). A randomised trial of nursing interventions supporting recovery of the postmastectomy patient. Journal of Clinical Nursing, 22(7/8), 919-929.

19. Chung, C. W., Hwang, E. \& Hwang, S. W. (2011). Details of Lymphedema, Upper Limb Morbidity, and Self Management in Women after Breast Cancer Treatment. Korean Journal of Women Health Nursing, 17(5), 474-483.

20. Dastan, N. B. \& Buzlu, S. (2012). Psychoeducation Intervention to Improve Adjustment to Cancer among Turkish Stage I-II Breast Cancer Patients: A Randomized Controlled Trial. Asian Pacific Journal of Cancer Prevention, 13(10), 5313-5318.

21. Dickerson, S. S., Alqaissi, N., Underhill, M. \& Lally, R. M. (2011). Surviving the wait: Defining support while awaiting breast cancer surgery. Journal of Advanced Nursing, 67(7), 1468-1479.

22. Dine, J. L., Austin, M. K. \& Armer, J. M. (2011). Nursing education on lymphedema self-management and self-monitoring in a South African oncology clinic. Journal of Cultural Diversity, 18(4), 126-128.

23. Elias, T.C., Mendes, L. C., Soares, M. B. 0. \& Silva, S. R. (2015). Characterization and functional capacity in women with breast cancer, gynaecological cancer and gestational trophoblastic disease. Revista Gaúcha de Enfermagem, 36(4), 37-42.

24. Haj, A. E. (2011). The effect of providing information to prevent lymphedema among treated breast cancer women. Middle East Journal of Nursing. Hashemite University, School of Medicine, 5(6), 16-18.

25. Haley-Emery, M. \& Schmitz-Johnson, W. (2014). Prospective protocol for lymphedema education and surveillance in a breast health center. Clinical journal of oncology nursing, 18, 27-31.

26. Husebø, A. M. L., Karlsen, B., Allan, H., Søreide, J. A. \& Bru, E. (2015). Factors perceived to influence exercise adherence in women with breast cancer participating in an exercise programme during adjuvant chemotherapy: A focus group study. Journal of Clinical Nursing, 24(3-4), 500-510.

27. Jeffs, E., Ream, E., Shewbridge, A., Cowan-Dickie, S., Crawshaw, D., Huit, M. \& Wiseman, T. (2016). Exploring patient perception of success and benefit in self-management of breast cancer-related arm lymphoedema. European journal of oncology nursing: the official journal of European Oncology Nursing Society, 20, 173-183.

28. Kimman, M. L., Dirksen, C. D., Voogd, A. C., Falger, P., Gijsen, B. C. M., Thuring, M., Lenssen, A., Ent, F. v. d., Verkeyn, J., Haekens, C., Hupperets, P., Nuytinck, J. K. S., Riet, Y. v., Brenninkmeijer, S. J., Scheijmans, L. J. E. E., Kessels, A. \& Boersma, L. J. (2011) Nurse-led telephone follow-up and an educational group programme after breast cancer treatment: results of a $2 \square 2$ randomised controlled trial. European journal of cancer (0xford, England: 1990), 47(7), 1027-1036.

29. Kuijpers, W., Groen, W. G, Loos, R., Oldenburg, H. S. A., Wouters, M. W. J. M., Aaronson, N. K., Hartenet, W. H. V. (2015). An interactive portal to empower cancer survivors: a qualitative study on user expectations. Supportive Care in Cancer, 23(9), 2535-2542.

30. Lee, H., Lim, Y., Yoo, M.-S., Kim, Y. (2011). Effects of a nurseled cognitive-behavior therapy on fatigue and quality of life of patients with breast cancer undergoing radiotherapy: an exploratory study. Cancer nursing, 34(6), E22-30. Consultado em Março, 2016, em http://www.ncbi.nlm.nih.gov/ pubmed/21372697.

31. Nizamli, F., Anoosheh, M. \& Mohammadi, E. (2011). Experiences of Syrian women with breast cancer regarding chemotherapy: A qualitative study. Nursing and Health Sciences, 13(4), 481-487.

32. Obeidat, R. F., Lally, R. M. \& Dickerson, S. S. (2012). Arab American women's lived experience with early-stage breast cancer diagnosis and surgical treatment. Cancer Nursing, 35(4), 302-311.
33. Obeidat, R. F., Dickerson, S. N., Homish, G. G., Alqaissi, N. M. \& Lally, R. M. (2013). Controlling Fear Jordanian Women's Perceptions of the Diagnosis and Surgical Treatment of Early-Stage Breast Cancer. Cancer Nursing, 36(6), 484-492.

34. Platt, J., Baxter, N., Jones, J., Metcalfe, K., Causarano, N., Hofer, S. 0. P., O'Neill, A., Cheng, T., Starenkyj, E. \& Zhong, T. (2013). Preconsultation educational group intervention to improve shared decision-making in postmastectomy breast reconstruction: study protocol for a pilot randomized controlled trial. Trials, 14, 199.

35. Ridner, S. H., Fu, M. R., Wanchai, A., Stewart, B. R., Armer, J. M. \& Cormier, J. N. (2012a). Self-Management of Lymphedema. Nursing Research, 61(4), 291-299.

36. Ridner, S. H., Bonner, C. M., Deng, J. \& Sinclair,V. J. (2012b). Voices from the shadows: living with lymphedema. Cancer Nursing, 35(1), E18-26. Consultado em Março, 2016 em http://www. pubmedcentral.nih.gov/articlerender.fcgi?artid=3172392\&tool=p mcentrez\&rendertype $=$ abstract

37. Rosenzweig, M., Brufsky, A., Rastogi, P., Puhalla, S., Simon, J. \& Underwood, S. (2011). The attitudes, communication, treatment, and support intervention to reduce breast cancer treatment disparity. Oncology nursing forum, 38(1), 85-89.

38. Runowicz, C. D., Leach, C. R., Henry, N. L., Henry, K. S., Mackey, H. T., Cowens-Alvarado, R. L., Cannady, R. S., Pratt-Chapman, M. L., Edge, S. B., Jacobs, L. A., Hurria, A., Marks, L. B., LaMonte, S. J., Warner, E., Lyman, G. H. \& Ganz, P. A. (2016). American Cancer Society / American Society of Clinical Oncology Breast Cancer Survivorship Care Guideline. CA-A Cancer Journal for Clinicians, 66(1), 43-73. Consultado em Março, 2016, em http:// onlinelibrary.wiley.com/doi/10.3322/caac.21319/full

39. Ryhänen, A. M., Rankinen, S., Tulusb, K., Korvenranta, H. \& LeinoKilpi, H. (2012). Internet based patient pathway as an educational tool for breast cancer patients. International Journal of Medical Informatics, 81(4), 270-278.

40. Sherman, K. A. \& Koelmeyer, L. (2011). The role of information sources and objective risk status on lymphedema risk-minimization behaviors in women recently diagnosed with breast cancer. Oncology Nursing Forum, 38(1), E27-E36. Consultado em Março, 2016, em http://www.scopus.com/inward/record.url?eid=2-s2.079953096391\&partnerID =40\&md5=0c104607befd6d52b00b7a 20dcb1f555

41. Sisman, H., Sahin, B., Duman, B. B, \& Tanriverdi, G. (2012). Nurseassisted education and exercise decrease the prevalence and morbidity of lymphedema following breast cancer surgery. Journal Of B.U.ON.: Official Journal Of The Balkan Union Of Oncology, 17(3), 565-569.

42. Spoelstra, S. L., Given, B. A., Given, C. W., Grant, M., Sikorskii, A., You, M. \& Decker, V. (2013). An Intervention to Improve Adherence and Management of Symptoms for Patients Prescribed Oral Chemotherapy Agents. Cancer nursing, 36(1), 18-28.

43. Tsuchiya, M.; Horn, S. \& Ingham, R. (2012). Information provision and problem-solving processes in Japanese breast cancer survivors with lymphoedema symptoms. Scandinavian Journal of Caring Sciences, 26(1), 53-60.

44. Ussher, J. M.; Perz, J. \& Gilbert, E. (2013). Information needs associated with changes to sexual well-being after breast cancer. Journal of advanced nursing, 69(2), 327-37.

45. Weaver, A., Love, S. B., Larsen, M., Shanyinde, M., Waters, R., Grainger, L., Shearwood, V., Brooks, C., Gibson, 0., Young, A. M. \& Tarassenko, L. (2014). A pilot study: dose adaptation of capecitabine using mobile phone toxicity monitoring - supporting patients in their homes. Supportive Care in Cancer, 22, 2677-2685.

46. Meijel, B., Gamel, C., Swieten-Duijfjes, B. \& Grypdonck, M. H. F. (2004). The development of evidence-based nursing interventions: Methodological considerations. Journal of Advanced Nursing, 48(1), 84-92.

47. Aranda, S. (2008). Designing nursing interventions. Collegian, 15, 19-25.

48. International Organization for Standardization. (2003). ISO 18104. Health informatics - Integration of a reference terminology model for nursing. Genebra: ISO.

49. International Council of Nurses. (2011). CIPE $囚$ Versão 2 Classificação Internacional para a Prática de Enfermagem. Lisboa: Ordem dos Enfermeiros.

50. Meleis, A. I., Sawyer, L. M., Im, E. O., Messias, H., D. K. Schumacher, K. (2000). Experiencing transitions: an emerging middle-range theory. ANS. Advances in nursing science, 23(1), $12-28$ 